\section{EINSTEIN AND RELATIVITY}

The Meaning of Relativity

By Albert Einstein. Fourth edition. Pp. v+146. (London : Methuen and Co., Ltd., 1950.) 7s. 6d. net.

HIS book is a new (the fourth) edition of Einstein's Stafford Little Lectures, delivered in May 1921 at Princeton University, supplemented by two appendixes, one on the "Cosmologic Problem", the other on the "Generalised Theory of Gravitation". It is the latter appendix which will attract the attention of physicists and mathematicians, because it contains the first complete account of Einstein's new attempt to establish by a priori reasoning the fundamental law of physics. But this appendix cannot be properly understood without a careful study of the whole book, and whatever the reader may think of the somewhat hazy view from the summit, he will enjoy the path which leads up to it. In fact, Einstein's presentation of the special and the general relativity is so simple, clear and convincing, that it can not only be recommended to students as a first introduction, but will also provide material for thought to scholars who are interested in the logical and empirical basis of the theory. The last chapter of the book ends with a consideration of Mach's ideas about the origin of inertia in connexion with the question of whether space is finite and closed, or infinite and open. This cosmologic problem is taken up in a wider frame in Appendix 1, which gives an account of Friedman's original theory. Later contributions to the theory of the expanding universe; the work of Lemaître, Robertson, Eddington, Milne and others, are not mentioned.

Appendix 2 is a confession of Einstein's deepest convictions about the structure of the material world, which have alienated him from the general trend of contemporary physics. He starts with the remark that the fundamental law of general relativity, which equates the contracted curvature tensor of spacetime to the energy tensor of matter, is unsatisfactory because of its dualistic character, and he proclaims his aim to establish a unitary theory, in which there exists only one field, subject to a law of the form : some generalization of the curvature tensor is zero. No separate energy tensor of matter ought to be introduced; it should later appear automatically if the new equations of the unitary field are split in two parts, one representing the action of inertia and gravitation, the other the rest of physical phenomena.

The natural way of obtaining the postulated generalization is, according to Einstein, the introduction of a metric tensor $g_{k l}$ which is not symmetrical. It can, of course, be split into a symmetric and skew-symmetric part, and it might be expected that the latter has something to do with the electromagnetic field. The objection may be raised that these asymmetric $g_{k l}$ are not an irreducible set of quantities, since each of the two parts is by itself a tensor. Einstein rejects this group-theoretical point of view as not being the only relevant one, and shows that a coherent tensor calculus based on the nonsymmetrical metric tensor can be developed and leads to reasonable results. Thus Einstein arrives at field equations of the formal type required. The main difficulty is the proof of the compatibility of the equations. The question now arises whether Einstein's equations fulfil his expectations of providing the foundations of a rational theory of matter. There is, however, no answer; the book ends with the sentence that these equations "form a system, of utmost simplicity, compatible in the usual sense". No solution and no discussion of the physical content are given.

What we have before us might therefore be better described as a programme than a theory. Einstein obviously hopes that his equations may have solutions which lead to the localization of energy, usually described as quanta, and to the laws of distribution of these quanta in agreement with wave mechanics.

The motive behind this programme is Einstein's well-known philosophical attitude. $\mathrm{He}$ believes that quantum mechanics is unsatisfactory, because it describes, not the behaviour of an objective world, but rather the reaction of the physical objects to the experimental conditions. Therefore he tries to find a theory of the classical type of such refined structure that it contains the essential features of atomistics. and quantum theory as consequences. There are at present few physicists who share this view. How ever, most of them will agree with Einstein that the final synthesis of relativity and quantum theory has yet to be made.

MAX BORN

\section{TOWN PLANNING}

\section{Cities in Evolution}

By Patrick Geddes. Edited by the Outlgok Tower Association, Edinburgh, and the Association for Planning and Regional Construction, London. New: and revised edition. Pp. xxxi + 241. (London: Williams and Norgate, Ltd., 1949.) 18s. net.

CITIES in Evolution" was first published in 1915, 1 just after the total loss of Geddes's first Town Planning Exhibition en route to India, and, apart from his earlier comprehensive study of the town planning of Dunfermline, undertaken at the request of Andrew Carnegie and published in 1903 under the title "City Development", it is the only book on civics and urban development that Geddes wrote. The book was intended to help the citizen to understand urban processes and to secure his active co-operation in urban development. Geddes's immediate influence was mainly direct and personal; but probably no one has done more to inspire the planning of cities and of regions to-day, and the reappearance of a volume which has so long been out of print should enable a new generation to judge for themselves how much the best elements in such planning owe to the thought of Geddes.

The present volume, however, is not simply a reprint of the original edition. Four chapters in that edition, those dealing with the homes of the people, the housing movement, a town-planning tour in Germany, and recent progress in housing and town planning, and all but two paragraphs of a chapter on German organisation have been omitted as being no longer of interest. Most of the deletions, which have been agreed between Dr. A. Geddes, Mr. Phipps Turnbull and Sir George Pepler, are fully justified, and any omissions are more than balanced by the inclusion of fresh material which gives the modern reader a clearer idea of the range of Geddes's social philosophy. The introduction includes an extract, dealing with the Valley Section, from a lecture given by Geddes at the New School of Social Research in New York City in 1923, which illustrates his use of the historical and geographical survey of occupations 